\title{
Using smartphones in cities to crowdsource dangerous road sections and give effective in-car warnings
}

\section{Mark D Dunlop}

Computer \& Information Sciences

University of Strathclyde, UK

Mark.Dunlop@strath.ac.uk

\section{Marc Roper}

Computer \& Information Sciences

University of Strathclyde, UK

Marc.Roper@strath.ac.uk

Mark Elliot

Psychology

University of Strathclyde, UK

Mark.A.Elliott@strath.ac.uk

Permission to make digital or hard copies of part or all of this work for personal or classroom use is granted without fee provided that copies are not made or distributed for profit or commercial advantage and that copies bear this notice and the full citation on the first page. Copyrights for third-party components of this work must be honored. For all other uses, contact the Owner/Author.

uses, contact the Owner/Author.

SEACHI 2016, May 08-08 2016, San Jose, CA, USA

SEACHI 2016, May 08-08 2016,

http://dx.doi.org/10.1145/2898365.2899796
Rebecca McCartan

sychology

Bruce McGregor

Psychology

University of Strathclyde, UK

bruce.mcgregor@strath.ac.uk

\begin{abstract}
The widespread day-to-day carrying of powerful smartphones gives opportunities for crowd-sourcing information about the users' activities to gain insight into patterns of use of a large population in cities. Here we report the design and initial investigations into a crowdsourcing approach for sudden decelerations to identify dangerous road sections. Sudden brakes and near misses are much more common than police reportable accidents but under exploited and have the potential for more responsive reaction than waiting for accidents. We also discuss different multimodal feedback conditions to warn drivers approaching a dangerous zone. We believe this crowdsourcing approach gives cost and coverage benefits over infrastructural smart-city approaches but that users need incentivized for use.
\end{abstract}

\section{Author Keywords}

Smart cities, smartphones, crowdsourcing, driving interfaces

\section{ACM Classification Keywords}

K.4.1 Public Policy Issues - Human safety 


\section{Introduction}

Road traffic crashes are a major cause of death worldwide, for example they accounted for over 300000 deaths in the South-East Asia Region during $2010^{1}$. In the United Kingdom, the Department for Transport catalogued over 145000 road accidents in 2012 that accounted for 1754 deaths [3]. Of these accidents approximately $75 \%$ took place on urban roads (defined by them as roads with a speed limit under $40 \mathrm{mph} / 64 \mathrm{kmh}^{-1}$ ). Motivated by these statistics and the work of Jun Rekimoto (e.g. [5]) we investigating the research questions: Can crowd sourcing techniques be used to identify dangerous road sections using standard smartphone accelerometers; and can multi-modal in-car feedback be used to affect driver behaviour in identified zones. In this extended abstract we present our studies into crowdsourcing of dangerous road junctions and different approaches to warn drivers on approach to dangerous junctions.

\section{Sudden brake detection approach}

Police data exists on accidents and road fatalities, however near misses are more common and if these can be identified they could result in more responsive understanding of dangerous road sections. To investigate whether crowd-sourcing techniques can be used to identify dangerous junctions using data from standard smartphone accelerometers, we developed Android-based detection code through a refinement process. Initially we tested in a control drive on an offroad skid-pan (Figure 1), then we carried out controlled drives through an quiet industrial estate simulating standard city driving and finally we recruited users who drove regularly around Glasgow and compared

\footnotetext{
${ }^{1}$ WHO: http://tinyurl.com/whoseasia-factsheet-pdf
}

identified hot-spots with historical police accident records. All studies were conducted under institutional ethical approval without smartphones while driving.

Our sudden-brake detection approach follows three steps: raw accelerations, smoothing and speed based filtering. We sample the overall strength of acceleration recorded by the phone (root square sum of $x, y$ and $z$ accelerometers). This prevents any orientation dependency with the phone so that users do not need to carefully place nor calibrate their phone. The samples are then smoothed to remove sensor noise. In the skid-pan trials we found that the sudden braking detectors could be triggered by fast increases in speed. Our first solution to this was to use a longer term orientation detection code to automatically adjust for the phone's orientation w.r.t. gravity. However, this was problematic as it led to variable sensitivity when the car was driven on steep hills. Instead we exploited GPS signal and filtered sudden accelerations that resulted in a noticeably reduced speed after the event. We are working on merging these two solutions.

To test sudden brake detection approach in a semirealistic but safe environment, we conducted a 45minute drive in a quiet industrial-estate road network in the evening when businesses were closed. The area was composed of with many junctions, some

roundabouts and variable quality road surface: all typical of many urban environments. We simulated nudging as if in line at a junction, drove over sections with speed bumps and conducted simulated emergency stops as well as normal driving. Three phones were tested in different locations: a MotoG (sitting in a cup holder), a Nexus5 (jacket pocket), and a S3Mini (glove box). The log of the journey is shown in Figure 2 . 


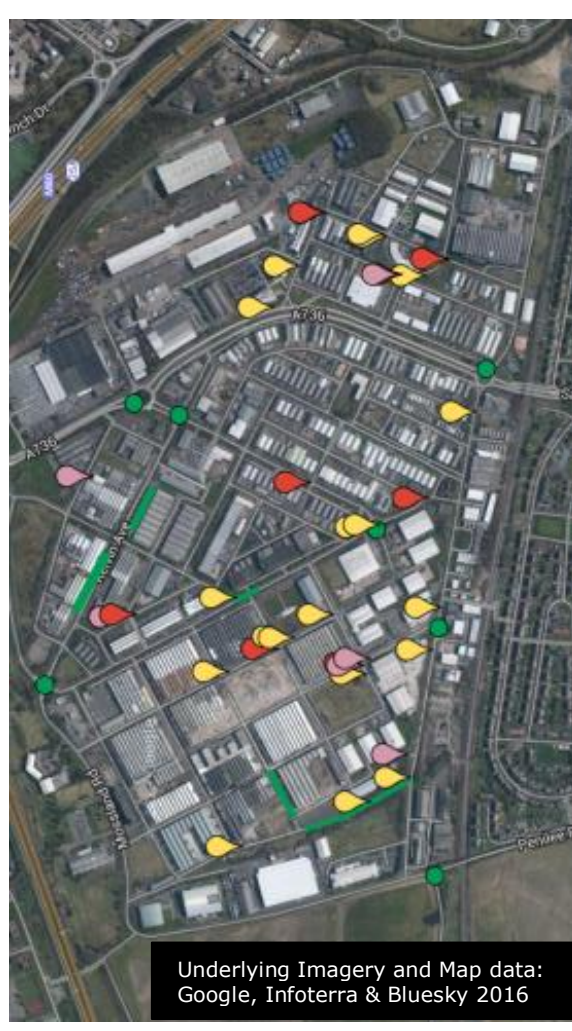

Figure 2. Manual log (red=sudden stops $/$ green $=$ potential false positive zones (bumps, roundabouts, etc.)) plus warnings from phones (yellow=S3Mini $/$ pink=Nexus5)
The correspondence between the sudden stops recorded and those identified by the phones is good but not perfect. The S3Mini generates many more warnings than the Nexus 5 which is likely because of its location (lose in the glove box). We are investigating techniques for tuning or limiting events, say to a fixed number per $100 \mathrm{~km}$. A graph of the logged accelerometer readings for the S3Mini and Nexus5 are shown in Figure 3 clearly showing much more activity on the lose phone. The MotoG (cup holder) showed strong correlation for the first part of the study with the Nexus5, however in the latter part of the journey the signal becomes fragmented due to the phone reducing sampling while asleep. Analysis of spikes from MotoG and Nexus5 showed strong correlation with manually recorded major braking events.

\section{Comparison with Police Data}

We recruited a small number of users to trial the system and recorded data for 12 days. Overall we recorded 2540 events which were grouped into 851 locations using distance between points clustering algorithms (simple and DBScan). Clusters had a mean number of events per location of 4.98 once we had filtered out locations with 1 or 2 triggers. Of the top 30 potentially most dangerous locations recorded for Glasgow by our system, we focused on the nine that were not on motorways ("urban roads" as defined by [3]). Each of these 9 examples was compared with data provided by ITOWorld ${ }^{2}$ that confirmed that all locations had multiple recent accidents. For example, our initial data recorded 6 sudden brakes at the location where Eglinton Street meets Bedford Street and similarly, 15 where it meets Cumbernauld Street (see Figure 5

ITO - Road Casualties UK - http://map.itoworld.com/ lower). In comparing this with the figure taken from ITO world showing previously recorded accidents (Figure 5 upper), it can be seen that our code has correctly identified the most concentrated accident points in the area. Unfortunately, one of the areas that the application identified has already proved to be fatal.

\section{Multimodal Driving Alerts}

Identifying dangerous road sections is only part of the problem. This data can be used to alter road designs but we were also interested to see if shorter term benefits could be gained by in-car warnings to slow drivers in dangerous zones. As well as improving in car safety this gives drivers the incentive to use the crowdsourcing app. We identified three modes of in-car alert: a spoken warning, an ambient red lighting effect and muting the music a driver is listening to. With the popularity of vocal notifications with in-car navigation systems, spoken alerts were an obvious modality to investigate. However, there has been some research showing spoken messages can interfere with short term memory in driving [8] and be ranked poorly by drivers for usability [1]. Listening to music while driving can improve mood and relax drivers, however it can also lead to less attentive driving $[4,2]$. As such we wanted to investigate if muting in-car audio could be used as an effective warning mechanism that increased awareness and slowed drivers. Finally, we wanted to investigate if ambient in-car red light could increase driver alertness based on the argument that warnings of medium urgency, for which visual modalities have been shown to be appropriate, can improve alertness when no critical event is present [6].

We ran studies in our driving simulator using drives lasting approximately 25 minutes / 20 km each through 

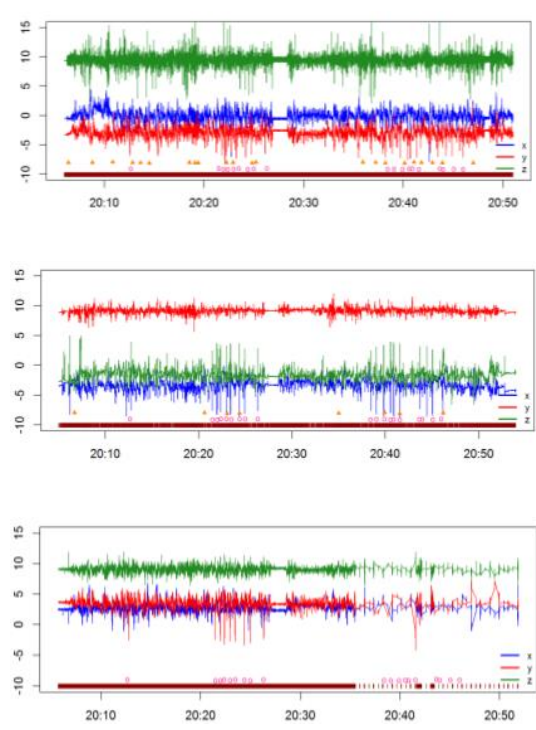

Figure 3 - S3Mini (top), Nexus5 and Moto $G$ accelerometer $\log (G \vee t)$

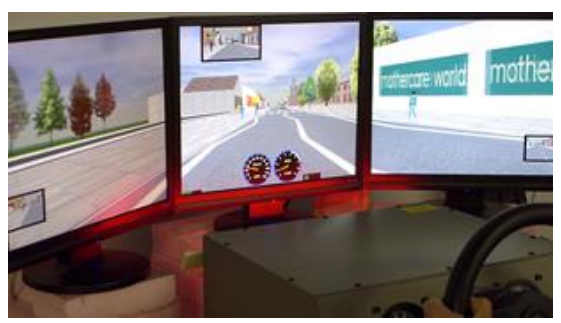

Figure 4 - Driving Simulator with ambient red lighting effect visible a city environment. Within these environments we coded 8 dangerous events: four instances each of pedestrians crossing the road ahead of the car and of crossing cars skipping the lights (other car driving across even though our vehicle had a green light). We used a three 19" monitor STISIM Drive M300W simulator (Figure 4) with physical steering wheel, indicators and pedals situated and operated as in a real car. Participants were asked to drive as if on an urban road in the real world and that the speed limit was 30 $\mathrm{mph}\left(48 \mathrm{kmh}^{-1}\right)$ and would remain at this throughout the route (there is reminder signage along the route). Ten junctions with traffic lights were programmed to be at red when the participants reached them, otherwise participants determined their own speed.

Our main study was structured around three pairwise tests - comparing control drives with one treatment per participant. In treatment conditions one of the mechanisms would be triggered approximately $150 \mathrm{~m}$ before the dangerous location and last for $150 \mathrm{~m}$ after the trigger location - generating a $300 \mathrm{~m}$ danger zone. All treatment warnings were triggered automatically by the simulator. Our initial analysis of results shows overall that speed is reduced significantly by $5 \%$ in danger zones with audio muting and slightly, but not significantly, with ambient lighting. This reduction did not appear to come at a cost of awareness (as measured by lane position variation) nor result in faster driving outside the danger zones. While audio muting led to only a $5 \%$ drop in speed we estimate this to result in a reduction of $17 \%$ in the likelihood of an impact resulting in death [7]. The verbal condition did not affect speed, the reasons need further investigation.

\section{Conclusions}

Our initial studies have shown that accelerometer data from smartphones can identify sudden brakes and that these align with known dangerous locations. This approach has the potential to identify dangers quicker and before accidents are reported on new or changed roads. Our simulator studies show that we can slow drivers using in-car feedback. We are now planning a wide-scale longitudinal deployment to evaluate the crowdsourcing more widely and gain user feedback on our MP3 player feedback mechanism in real city driving.

\section{Acknowledgements}

We are grateful to our colleague Neil Ferguson for input into the project. The code has been largely developed in student projects, in particular our thanks to Jonathan Innes, Ryan McNulty, John Valenti, Gabriel GiordanoBatista, Anderson Queiroz, Christopher Sloey and Igor Calabria Da Fonte for their development work. We are also grateful to the participants of our alert study and those who participated in earlier test drives. Part of this research was funded through Strathclyde's EPSRC funded Bridging the Gap programme.

\section{References}

1. D Beattie, L Baillie, and M Halvey. 2015. A comparison of artificial driving sounds for automated vehicles. Proc Ubicomp 2015, ACM, 451-462.

2. W Brodsky. 2001. The effects of music tempo on simulated driving performance and vehicular control. Transportation Res. F: Traffic Psych. \& Beh.. 4(4).

3. Department for Transport. Road Accident Statistics Branch. 2013. Road Accident Data 2012. UK Data Archive. $d x$.doi.org/10.5255/UKDA-SN-7431-1 




Figure 5: Eglinton Street Comparison
4. N Dibben and V J Williamson. 2007. An exploratory survey of in-vehicle music listening. Psychology of Music 35, 4: 571-589.

5. T Miyaki \& J Rekimoto. 2008. Sensonomy: Envisioning Folksonomic Urban Sensing. UbiComp workshop.

6. I Politis, SA Brewster \& F Pollick. 2014. Evaluating multimodal driver displays under varying situational urgency. Proc SIGCHI., ACM, 4067-4076.
7. E Rosen \& U Sander. 2009. Pedestrian fatality risk as a function of car impact speed. Accident Analysis \& Prevention 41, 3: 536-542.

8. R Vilimek \& T Hempel. 2005. Effects of speech and non-speech sounds on short-term memory and possible implications for in-vehicle use, Proc. ICAD. 